\title{
ESTUDO MORFOLOGICO DA LARVA DE ÚLTIMO ÍNSTAR DE MIATHYRIA SIMPLEX (RAMBUR) (ODONATA, LIBELLULIDAE)
}

\author{
J. M. Costa $^{1}$ \\ C. V. de Assis ${ }^{1}$
}

\begin{abstract}
MORPHOLOGICAL STUDY OF THE LAST INSTAR LARVAE OF MIATHYRIA SIMPLEX (RAMBUR) (ODONATA, LIBELLULIDAE). The larva of Miathyria simplex (Rambur, 1842) is described, illustrated and compared to that of M. marcella (Selys, 1857). A key for identification of the South American Libellulidae larvae, wich show dorsal spines on abdominal segments III to VIII, is presented.

KEY WORDS. Miathyria simplex, Libellulidae, Odonata, neotropical, larvae, description
\end{abstract}

O gênero Miathyria Kirby, 1889 inclue duas espécies: M. marcella (Selys, 1857), com registro na América do Sul, América Central e Sul dos Estados Unidos e M. simplex (Rambur, 1842), com registro na América do Sul e América Central (DAVIES \& TOBIN, 1985; SANTOS, 1981, 1988).

A larva de $M$. marcella, descrita pela primeira vez por BICK (1953, fevereiro) e posteriormente por WESTFALL (1953, março) apresenta similaridade com a larva de $M$. simplex que LIMONGI (1990) descreveu, junto a larvas de Libellulidae de cinco outras espécies da Venezuela.

Neste trabalho é descrita a exúvia da larva de último estádio de $M$. simplex, comparando-a com as exúvias de último estádio de $M$. marcella depositadas na Coleção Entomológica do Museu Nacional, Universidade Federal do Rio de Janeiro e com as descrições anteriores. Uma chave para identificação da larvas de Libellulidae da América do Sul que apresentam espinhos dorsais dos segmentos abdominais III ao VIII, é fornecida.

\section{Miathyria simplex (Rambur, 1842)}

Larva de tamanho pequeno, coloração geral ocrácea (Fig. 1).

Cabeça tão larga quanto o tórax; olhos compostos arredondados lateralmente, projetados posteriormente atingindo $2 / 3$ do comprimento médio-dorsal da cabeça; antenas com sete artículos (Fig. 2), sendo os três últimos guarnecidos por pêlos finos e longos; mandíbulas (Fig. 7) com dentes incisivos obtusos,

1) Departamento de Entomologia, Museu Nacional, Universidade Federal do Rio de Janeiro, Quinta da Boa Vista, São Cristóvão, 20940-040 Rio de Janeiro, Rio de Janeiro, Brasil. Bolsista do CNPq. 

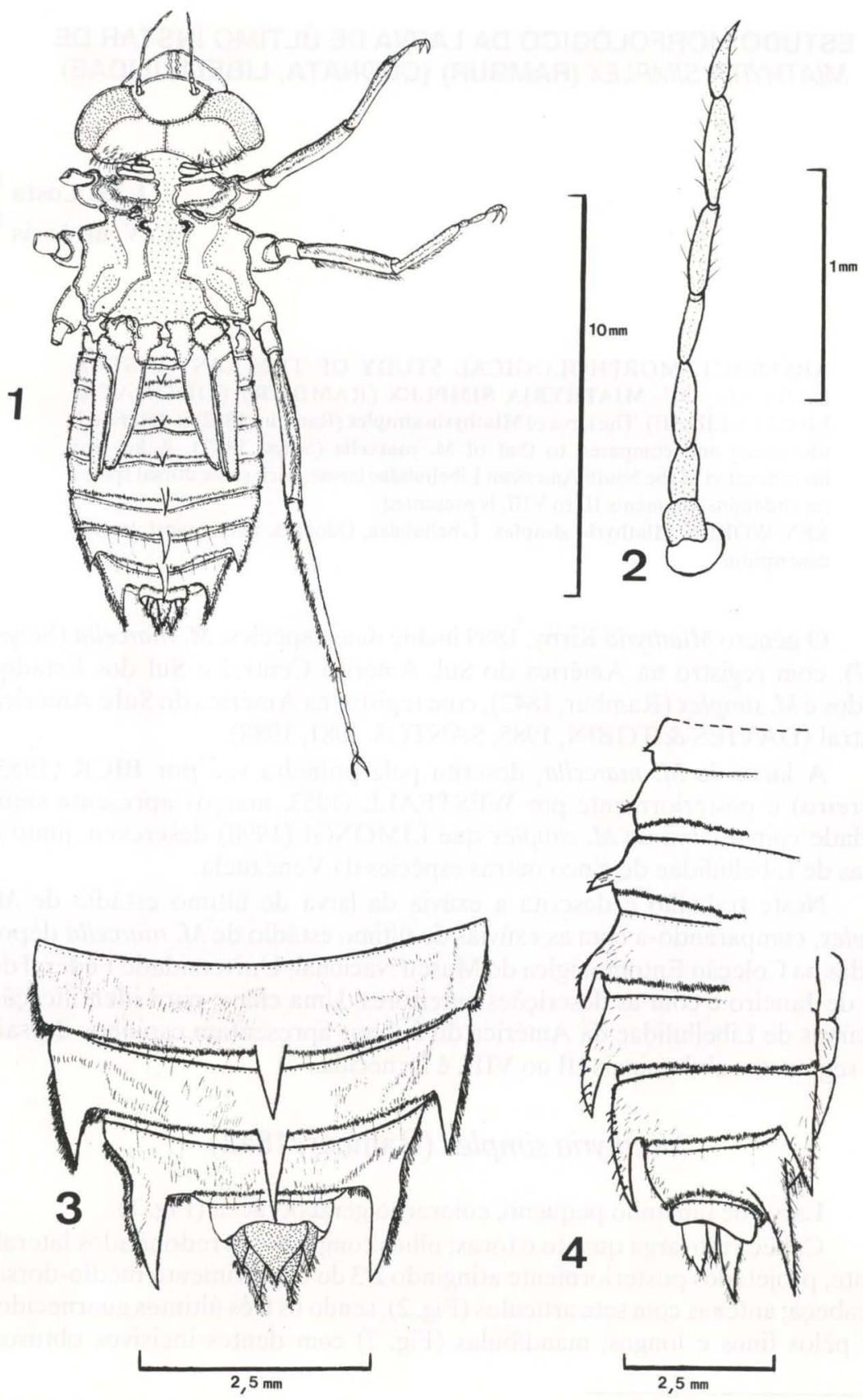

Figs 1 - 4. Miathyria simplex (Rambur). (1) Exúvia da larva de último ínstar; (2) antena esquerda; (3) vista dorsal dos últimos segmentos abdominais; (4) vista lateral do abdome. 
dentes molares de forma irregular; fórmula mandibular (de acordo com WATSON, 1956) $\frac{l}{r} \frac{1234}{1234} \frac{o}{y} \frac{a b d}{a b d}$. Lábio largo, quando dobrado alcançando o nível do segundo par de pernas. Pré-mento (Fig. 5) com um par de 11 setas; margem distal com uma série de 12 setas espiniformes de cada lado e quatro apicais; superfície interna com uma série de 36 pequenas cerdas transparentes, duas pequenas e robustas setas em cada lado da margem lateral, na conexão com os palpos labiais. Palpo labial (Fig. 8) largo, com pequenas máculas castanho-escuras; seis setas em cada palpo; parte basal com nove setas espiniformes; garra móvel ligeiramente menor que a seta proximal; margem distal (Fig. 6) com nove crenulações suaves, cada uma suportando de três a quatro setas; número de setas em cada crenulação, partindo da garra móvel: 3, 3, 4, 3, $3,4,4,3,3$; margem interna com 19 setas irregularmente distribuídas; margem externa lisa.

Tórax com processo supracoxal protorácico pequeno com bordo retilíneo. Tecas longas armadas de fileiras de espinhos e cerdas.

Abdome cilíndrico, mais alargado no VI segmento; margem externa provida de pequenas cerdas concentradas a partir da metade do VII segmento (inclusive nos espinhos laterais VIII e IX) (Fig. 3); superfície dorsal com longos pêlos dispersos nas margens posteriores dos segmentos VII, VIII e IX; espinhos dorsais presentes do III ao VIII segmentos com as seguintes características: III e IV curtos, originando-se no meio dos segmentos; V e VI curvos e robustos, originando-se na extremidade distal; VII e VIII afilados, originando-se aproximadamente no bordo posterior dos segmentos; espinho do VII segmento atingindo $2 / 3$ do comprimento médio-dorsal do VIII segmento; espinho do VIII segmento ultrapassando a margem anterior do IX segmento; providos de numerosas setas. Espinhos laterais presentes nos segmentos VIII e IX; espinho do VIII segmento reto e estendendo-se até $2 / 3$ do comprimento médio-dorsal do IX segmento; espinho do IX segmento levemente curvado e pouco mais de duas vezes maior que o comprimento médio-dorsal do IX segmento, estendendo-se até a extremidade lateral do apêndice abdominal (Fig. 4); epiprocto largo na base e fortemente afilado na extremidade; paraprocto distintamente maior que o epiprocto e levemente curvado para baixo distalmente; cerco atingindo aproximadamente $2 / 3$ do comprimento do epiprocto, levemente curvado para baixo distalmente. Todos providos de pêlos nas extremidades.

Material examinado. Brasil, Rio de Janeiro, Rio de Janeiro, Braço do Rio São João (CEDAE), 18.VI.1981 (emergência 4.VIII.1981), um macho, N.D. Santos \& L.F. Netto leg; Mini-Horto Sylvio Potsch (Colégio Pedro II, São Cristóvão, Rio de Janeiro), 8.IV.1986 (emergência 31.V.1986), duas fêmeas, J.R. Pujol leg; material depositado na Coleção de Entomologia do Departamento de Entomologia do Museu Nacional, Universidade Federal do Rio de Janeiro.

Medidas em milímetros. Comprimento total 14,1; comprimento e largura máxima da cabeça 3,0:4,8; comprimento e largura máxima dos olhos 2,3:1,28; comprimento das antenas 2,3; comprimento relativo dos antenômeros 33:21: 


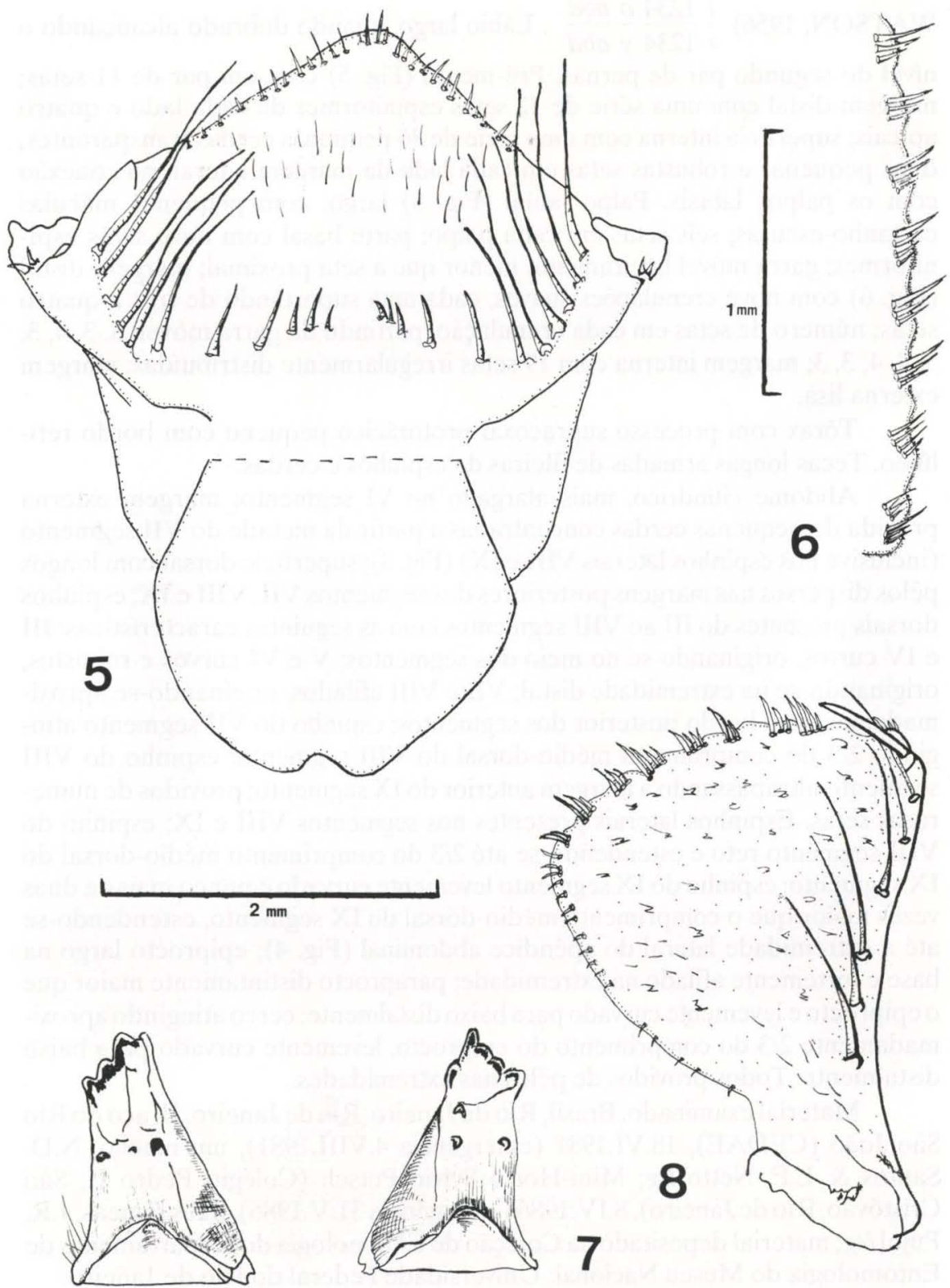

Figs 5 - 8. Miathyria simplex (Rambur). (5) Pré-mento em vista dorsal; (6) margem distal do palpo labial direito, mostrando em detalhe as crenulações; (7) superfície interna das mandibulas esquerda e direita; (8) palpo labial direito em vista dorsal. 
33:27:36:44:36; comprimento, largura basal e largura máxima do pré-mento 4,35:1,99:3,52; comprimento do palpo labial 2,47; comprimento da teca alar anterior e posterior 4,32:4,72; comprimento dos fêmures I, II e III 2,47:3,36:4,48; comprimento das tíbias I, II e III 2,40:2,96:4,32; comprimento e largura máxima do abdome 7,65:4,65; comprimento e largura basal do espinho lateral VIII segmento 0,59:0,25; comprimento e largura basal do espinho lateral IX segmento $1,00: 0,34$; epiprocto 0,63 ; paraprocto 0,98 ; cerco 0,50 .

\section{DISCUSSÃO E CONCLUSÕES}

Todos os caracteres apresentados por BICK (1953) para a larva de $M$. marcella foram observados nos exemplares coletados no Estado do Rio de Janeiro. Os caracteres apresentados por WESTFALL (1953) para esta mesma espécie não foram coincidentes com os de nossos exemplares no que se refere ao número de setas nas crenulações do palpo labial e no espinho abdominal dorsal. Segundo WESTFALL (op.cit.), somente alguns exemplares apresentam espinho dorsal no III segmento abdominal. No material analisado (exúvias de último ínstar) M. marcella e $M$. simplex apresentam espinho dorsal do III ao VIII segmentos abdominais. Em relação ao número de setas nas crenulações, na figura de WESTFALL não foi possível observar o número de setas na primeira crenulação, entretanto, foi possível estabelecer o número de setas em cada uma das nove crenulações ( o autor cita dez crenulações) começando da garra móvel: $7,3,4,5,4,2,3,6,4,2$.

Os exemplares observados, apresentam os mesmos números de setas em cada crenulação estabelecido por BICK (1953): 2, 3, 4, 5, 4, 3, 5, 4, 4, 3. O exemplar de Cuba, mencionado por WESTFALL (1953) é considerado neste trabalho como $M$. simplex. Face às diferenças entre os exemplares de $M$. marcella examinados e à descrição de WESTFALL, acredita-se que no material por ele analisado, houvesse exemplares de $M$. marcella, porém, a descrição e o desenho provavelmente tiveram como suporte exemplares não pertencentes a este gênero, uma vez que Miathyria possui espinho dorsal do III ao VIII segmentos abdominais.

A larva de $M$. simplex redescrita aqui diferencia-se da descrita por LIMONGI (1990) por apresentar os últimos cinco antenômeros de tamanho diferentes: $7=36,6=44,5=36,4=27,3=33$; setas pré-mentonianas: $11 \mathrm{em}$ cada lado; margem distal do palpo labial com nove crenulações; número de cerdas em cada crenulação, começando da garra móvel: 3, 3, 4, 3, 3, 4, 4, 3; margem interna do palpo labial com 19 cerdas e espinho dorsal do III ao VIII segmentos abdominais. $\mathrm{O}$ autor analisa nove exemplares, todos da mesma localidade, afirmando possuir oito exemplares com espinho dorsal do IV ao VIII segmentos abdominais. ${ }^{\mathrm{Na}}$ figura da larva de último ínstar, é apresentado o exemplar com espinho dorsal do III ao VIII segmentos abdominais. As figuras 65,66 e 70 são coincidentes com as figuras 1,3 e 4 aqui apresentadas. É possível que o único exemplar de $M$. simplex analisado pelo autor seja aquele que possui espinhos dorsais do III ao VIII segmentos abdominais, ou ainda, que o espinho 
do III segmento abdominal dos outros oito exemplares fosse tão pequeno que o autor não tenha observado.

Das larvas de Libellulidae da América do Sul conhecidas, Elasmothemis Westfall, 1988, Macrodiplax Brauer, 1868, Miathyria Kirby, 1889 e Libellula Linnaeus, 1758, são os únicos gêneros cujas larvas de último ínstar apresentam espinhos dorsais do III ao VIII segmentos abdominais (BICK, 1955; DE MARMELS, 1982; PUJOL-LUZ \& COSTA, 1987; WESTFALL, 1988; PUJOL-LUZ, 1990). DAVIES \& TOBIN (1985) registraram Libellula croceipennis, Selys, 1868 na América do Sul e Sul dos Estados Unidos, entretanto, não encontramos qualquer registro desta espécie na América do Sul.

A larva de M. marcella é freqüente nos mais variados ambientes lênticos, onde pode ser encontrada em grande quantidade, o mesmo não ocorrendo com a larva de M. simplex. Embora as duas espécies convivam nos mesmos ambientes, a predominância de $M$. marcella sobre $M$. simplex é facilmente comprovada pelo número de exemplares coletados.

As larvas destas duas espécies já foram coletadas junto com as larvas de Acanthagrion cuneatum Selys, 1876, Diastatops obscura (Fabricius, 1775), Epipleoneura venezuelensis Racenis, 1955, Erythemis mitroides (Brauer, 1900), Erythemis plebeja (Burmeister, 1839), Neoneura bilinearis Selys, 1860, Erythrodiplax connata fusca (Rambur, 1842) e Miathyria hesperis Ris, 1911.

Chave para identificação de larvas das espécies de Libellulidae da América do Sul que apresentam espinho dorsal do III ao VIII segmentos abdominais

1. Espinhos laterais nos segmentos VI ao IX ......... Elasmothemis . . 4

- Espinhos laterais somente nos segmentos VIII e IX ............... 2

2. Presença de seis ou sete setas palpais em cada lobo; setas pré-mentais variando entre dez e $12 \ldots \ldots \ldots \ldots \ldots \ldots \ldots$. Miathyria $\ldots .6$

- Presença de nove a 12 setas palpais em cada lobo; setas pré-mentais variando

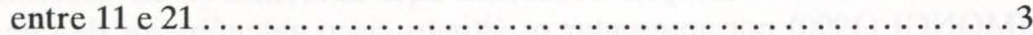

3. Presença de 11 a 12 setas palpais em cada lobo; setas pré-mentais variando entre 18 e $21 \ldots \ldots \ldots \ldots \ldots \ldots \ldots \ldots \ldots \ldots$ Macrodiplax (M. balteata)

- Presença de nove a dez setas palpais em cada lobo; setas pré-mentais variando

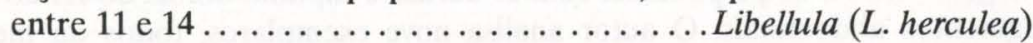

4. Espinho lateral do IX segmento abdominal não alcançando a extremidade do paraprocto; espinho dorsal do VIII segmento abdominal visivelmente menor que o comprimento dos espinhos anteriores e não alcançando o IX segmento .............................. williamsoni

- Espinho lateral do IX segmento abdominal ultrapassando a extremidade do 


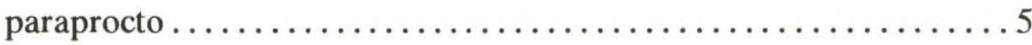

5. Espinho lateral do IX segmento abdominal tão longo quanto o comprimento médio-dorsal do mesmo segmento; espinhos dorsais do V ao VII segmentos abdominais dobrados em forma de gancho; nove a dez setas no pré-mento .................................nnacrioides

- Espinho lateral do IX segmento abdominal duas vezes maior que o comprimento médio-dorsal do mesmo segmento; espinhos dorsais do III ao VII segmentos abdominais dobrados em forma de gancho; 11 a 13 setas no pré-mento ............................. constricta

6. Seis setas palpais; espinho dorsal do VII segmento abdominal alcançando $2 / 3$ do comprimento médio-dorsal do VIII segmento; espinho dorsal do VIII segmento abdominal ultrapassando o IX segmento; comprimento total variando entre 13 e $14 \mathrm{~mm} . . \ldots \ldots \ldots \ldots \ldots \ldots \ldots \ldots \ldots$ simplex

- Sete setas palpais; espinho dorsal do VII segmento abdominal alcançando 1/3 do comprimento médio-dorsal do VIII segmento; espinho dorsal do VIII segmento abdominal não ultrapassando o IX segmento; comprimento total variando entre $16 \mathrm{e} 17,5 \mathrm{~mm} \ldots \ldots \ldots \ldots \ldots \ldots \ldots$ marcella

AGRADECIMENTOS. Agradecemos aos Professores José Roberto Pujol-Luz e Alcimar do Lago Carvalho, da Universidade Federal do Rio de Janeiro, pelas valiosas sugestões.

\section{REFERÊNCIAS BIBLOGRÁFICAS}

BICK, G.H. 1953. The nymph of Miathyria marcella (Selys) (Odonata, Libellulidae). Proc. Ent. Soc. Washington 55: 30-36.

1955. The nymph of Macrodiplax balteata (Hagen). Proc. Ent. Soc. Washington 57 (4): 191-196.

DAVIES, D.A.L. \& P. TOBIN. 1985. The dragonflies of the world: a systematic list of the extant species of Odonata. 2. Anisoptera. Soc. Int. Odonatol. 5: 1-151.

DE MARMELS, J. 1982. Cuatro nayades nuevas de la familia Libellulidae (Odonata: Anisoptera). Bol. ent. venez. N.S. 2 (11): 94-101.

LIMONGI, J.E. 1990. Estudio morfo-taxonomico de nayades de algunas especies de Odonata (Insecta) en Venezuela (II). Mem. Cienc. Nat. La Salle 133: 405-420.

PUJOL-LUZ, J.R. \& J.M. COSTA. 1987. Descrição da larva de Dythemis cannacrioides Calvert, 1906 (Odonata: Libellulidae). Atas Soc. Biol. Rio de Janeiro 27: 19-10.

PUJOL-LUZ, J.R. 1990. Descrição da larva de Elasmothemis constricta (Calvert, 1898) (Odonata: Libellulidae). Rev. Bras. Biol. 50 (2): 487-490.

SANTOS, N.D. 1981. Odonata, p. 64-85. In: S.H. HULBERT; G. RODRIGUEZ \& N.D. SANTOS (ed.) Aquatic biota od Tropical South 
America. Part I. Arthropoda. San Diego, San Diego State University.

- 1988. Catálogo bibliográfico de ninfas de odonatos neotropicais. Acta Amazon. 18 (1-2): 265-350.

WATSON, M.C. 1956. The utilization of mandibular armature in taxonomic studies of anisopterous nymphs. Trans. Amer. Ent. Soc. 81: 155-202.

WESTFALL, M.J. JR. 1953. The nymph of Miathyria marcella (Selys) (Odonata). Florida Ent. 36: 21-25.

- 1988. Elasmothemis gen. nov., a new genus related to Dythemis (Anisoptera, Libellulidae). Odonatologica 17 (4): 419-428.

Recebido em 04.Il.1993; aceito em 20.IX.1993. 